\title{
Self-Healing of Interfacial Debonding in Fiber-Reinforced Polymers and Effect of Microstructure on Strength Recovery
}

\author{
K. Sanada ${ }^{*}, 1$ N. Itaya ${ }^{1}$ and Y. Shindo ${ }^{2}$ \\ ${ }^{1}$ Department of Mechanical Systems Engineering, Toyama Prefectural University, Kurokawa 5180, Imizu, Toyama 939- \\ 0398, Japan \\ ${ }^{2}$ Department of Materials Processing, Graduate School of Engineering, Tohoku University, Aoba-yama 6-6-02, Sendai \\ 980-8579, Japan
}

\begin{abstract}
This study focuses on the optimizing the microstructure to improve the efficiency for healing interfacial debonding in fiber-reinforced polymers (FRPs). Healing is accomplished by incorporating a microcapsulated healing agent and catalytic chemical trigger within a coating layer on the surface of the fiber strands. Self-healing is demonstrated on flat tensile specimens of unidirectional FRPs. The effects of microcapsule diameter and concentration, and number of filaments in the fiber strand on tensile strength of virgin and healed specimens are discussed. Microstructure of the fracture surfaces of specimens was also examined by a scanning electron microscope. Additionally, finite element analyses were performed to predict the microcapsule-matrix debonding process during uniaxial tensile loading.
\end{abstract}

\section{INTRODUCTION}

The interface between matrix and fiber has a major influence on the mechanical properties of fiber-reinforced polymers (FRPs). Damage at the interface such as fibermatrix debonding can cause a reduction in the undamaged structural strength and stiffness of FRPs. As a result, the structural capability of the FRP is reduced, and premature failure can result if the damage is not detected and repaired. However, microscopic damage such as interfacial debonding is extremely difficult to detect and repair by conventional methods. The current research suggests that repair of microscopic damage can be accomplished by incorporating repair components into the FRP. This novel concept is that of self-healing.

The idea of a self-healing material has led to significant interest in the current literature. Many techniques have focused on the ability to heal internal damage in FRPs. Hayes et al. [1] reported on the optimization of a solidstate self-healing resin system and its subsequent use as a matrix for polymer composites. Trask et al. [2] investigated self-healing using hollow fibers embedded within both glass/epoxy and carbon/epoxy laminates. Similar approaches were adopted by Dry et al. [3], Motuku et al. [4], Bleay et al. [5] and Pang et al. [6]. White et al. [7] developed a self-healing polymer with a micro-encapsulated healing agent. The healing agent was dicyclopentadiene (DCPD) monomer. Kessler and colleagues [8] reported on initial studies using the self-healing polymer as the matrix material of woven glass/epoxy laminates and discussed the self-healing of interlaminar fracture (delamination). Yin et al. [9] studied self-healing woven glass/epoxy laminates with epoxy-filled microcapsules. Recently,

*Address correspondence to this author at the Department of Mechanical System Engineering, Toyama Prefectural University, Kurokawa 5180, Imizu, Toyama 939-0398, Japan; Tel: +81-766-56-7500; Fax: +81-76656-6131; E-mail: sanada@pu-toyama.ac.jp
Sanada et al. [10] proposed a methodology for self-healing of interfacial debonding in unidirectional carbon/epoxy composites by using fiber strands coated with the self-healing polymer developed by White et al.

The scope of this work is to optimize numerically and experimentally the microstructure to improve the performance of this self-healing system as shown in Fig. (1). Transverse tensile tests were carried out with flat tensile specimens of unidirectional FRPs. The influences of microcapsule diameter and concentration, and number of filaments in the fiber strand on the tensile strength of virgin and healed specimens are examined. Post-fracture specimens were also examined by a scanning electron microscope (SEM) to study the microstructure. In addition to conducting experiments, finite element analyses were employed to study the microcapsule-matrix debonding process during uniaxial tensile loading.

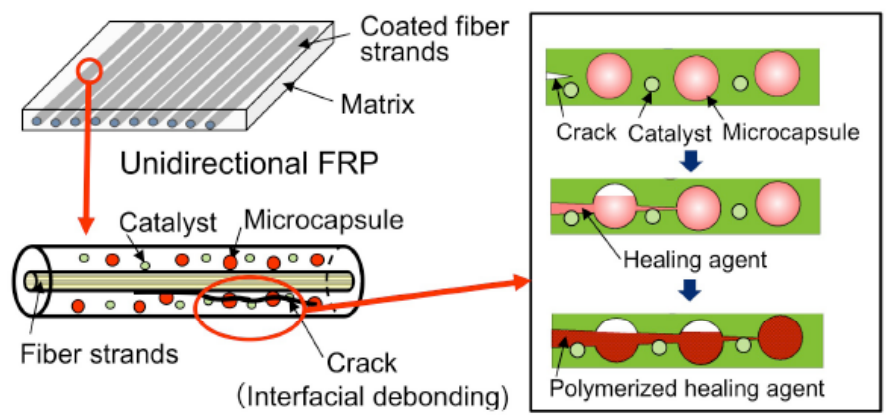

Fig. (1). Self-healing system of interfacial debonding.

\section{EXPERIMENTAL PROCEDURES}

\section{Materials and Specimen Fabrication}

Self-healing FRP plates made of unidirectional carbon fiber-reinforced polymers were prepared. Torayca T300B (Toray Industries, Inc.) carbon fiber strands were coated by manually dipping them into the Epikote 828 (Japan Epoxy 
Resins Co. Ltd) /Ancamine K54 (Air Products and chemicals, Inc.) epoxy mixture containing DCPD-filled microcapsules and $2.5 \mathrm{wt} \%$ Grubbs catalyst (Sigma-Aldrich Co.). DCPD was encapsulated in a urea-formaldehyde polymer shell via our previously reported method [10]. The number of filaments $N$ in the fiber strand was 3000, 6000 and 12000. The mean diameters $d$ of the microcapsules were around 50,100, 200, 300 and $400 \mu \mathrm{m}$ and the microcapsule concentration $W_{f}$ was varied from 10 to $40 \mathrm{wt} \%$. After coating, the fiber strands were held straight and cured for $24 \mathrm{~h}$ at room temperature in order to obtain proper hardness.

The semi-cured fiber strands were placed in mold and impregnated with the Epikote 828 (Japan Epoxy Resins Co. Ltd)/diethylenetriamine (DETA) epoxy resin. The coated fiber strands were aligned perpendicular $\left(90^{\circ}\right.$ direction) to the long direction of the plate and the fiber volume fraction was set to be about $3 \mathrm{vol} \%$. The mold was then closed and the samples were molded into the plate for $24 \mathrm{~h}$ at room temperature, followed by $24 \mathrm{~h}$ at $40^{\circ} \mathrm{C}$. Once the plates were cured, they were machined using a water cooled diamond saw and a milling machine to produce flat tensile specimens as shown in Fig. (2). Specimens had a nominal thickness of $3 \mathrm{~mm}$ and a gauge length of $25 \mathrm{~mm}$.

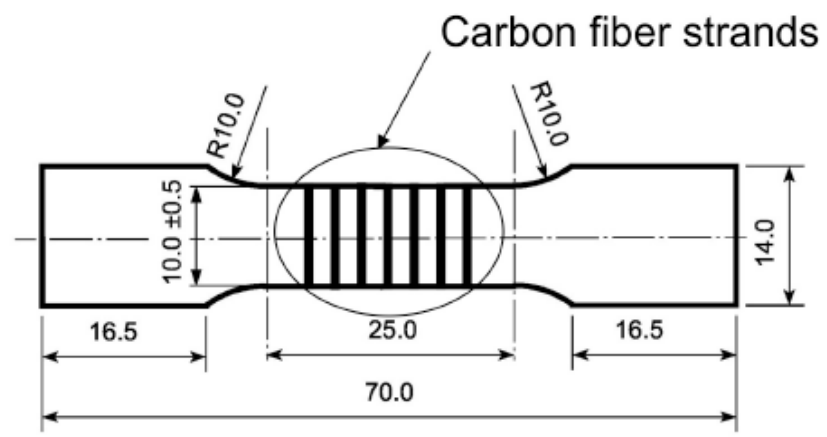

Fig. (2). Specimen geometry (dimensions in $\mathrm{mm}$ ).

\section{Test Methods}

The specimens were tested using a tensile test machine in the displacement control at rate of $0.5 \mathrm{~mm} / \mathrm{min}$. Six specimens were tested for each data point. Scatter of the data is indicated by error bars in the figures. The test procedure was as follows. First, virgin specimens were loaded to failure. After failure, the specimens were unloaded, clamped closed, and allowed to heal for 10 days at $30^{\circ} \mathrm{C}$. After healing, the specimens were reloaded to failure again.

Healing efficiency $\eta$ is defined as the ability of a healed specimen to recover transverse tensile strength

$\eta=\frac{\sigma_{c}^{\text {healed }}}{\sigma_{c}^{\text {virgin }}}$

where $\sigma^{\text {virgin }}$ is the tensile strength of the virgin specimen and $\sigma_{c}^{\text {healed }}$ is the tensile strength of the healed specimen.

SEM observations were performed to investigate the fracture surfaces of the healed specimens. Fracture surfaces were prepared for analysis by sputter coating with goldpalladium.

\section{FINITE ELEMENT ANALYSIS}

In previous work on this self-healing system [10], an investigation of the healed fracture planes revealed that many microcapsules fractured with protruding shell materials representative of debonding. As the microcapsule-matrix debonding occurs, the release of healing agent from the microcapsules is believed to be incomplete. This leads to incomplete coverage of the healing agent on the fracture plane and a lowering of the healing efficiency. Thus, we investigated the effect of microstructure on the microcapsule-matrix debonding behavior in order to achieve high healing efficiency.

The 3D unit cell model is shown in Fig. (3). The form of this unit cell model is based on the assumption of a uniform and periodic distribution of microcapsules. The origin of the coordinates coincides with the center of the unit cell. Due to the symmetry of periodic packing, it is sufficient to analyze one-eighth of the cubic matrix. The diameter of the spherical microcapsule is taken as $2 R$, while the size of the cubic matrix is denoted as $2 L$. The thickness of the microcapsule membrane is $t$. A spherical membrane is filled with an incompressible fluid (healing agent) and is inflated by internal pressure $p$. The microcapsule volume fraction $V_{f}$ is given by

$$
V_{f}=\frac{\pi}{6}\left(\frac{R^{3}}{L^{3}}\right)
$$

Symmetry conditions are applied to the boundary surfaces $x_{1}=0, x_{2}=0$ and $x_{3}=0$. The boundary conditions applied to surface $x_{1}=L$ and $x_{2}=L$ should enforce periodicity along the $x_{1}$ and $x_{2}$ directions. The top surface $x_{3}=L$ is subject to a uniform displacement $u^{*}$ in the $x_{3}$ direction.
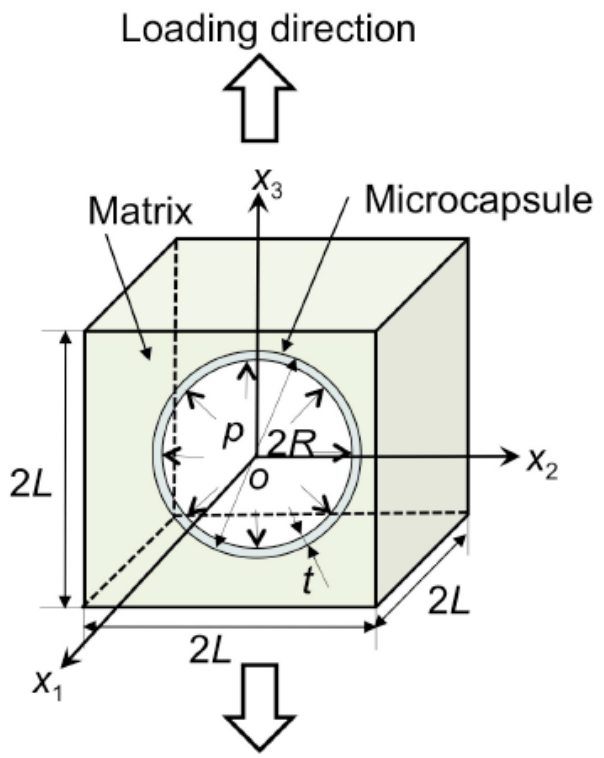

Fig. (3). Schematic diagram of a unit cell model.

The individual phase materials are modeled as linear elastic and isotropic solids. For the microcapsule membrane, the Young's modulus and the Poisson's ratio are assumed to be $1 \mathrm{GPa}$ and 0.3 , respectively. The Young's modulus and the Poisson's ratio of the epoxy matrix are taken to be $3 \mathrm{GPa}$ and 0.35 [11], respectively. 
In this analysis, it is assumed that mode I debonding occurs at interface between matrix and microcapsule. A bilinear cohesive zone model [12] is adopted to model the debonding behavior of the interface. As shown in Fig. (4), this model is governed by the maximum normal traction $\sigma_{\max }$ that corresponds to the interfacial strength, and $\delta_{c n}$, which denotes the critical value of the normal displacement jump, characterizing the complete failure of material. Therefore, the critical fracture energy $G_{c n}$ is determined by:

$G_{c n}=\frac{1}{2} \delta_{c n} \sigma_{\max }$

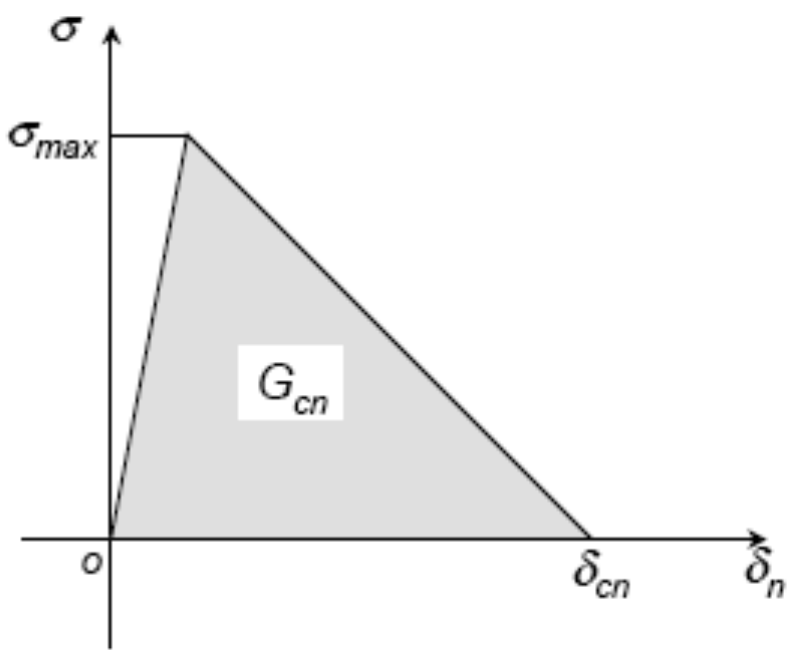

Fig. (4). Cohesive law for mode I fracture.

\section{RESULTS AND DISCUSSIONS}

\section{Transverse Tensile Test}

The virgin and healed tensile strengths $\left(\sigma_{c}\right)$, and healing efficiency $(\eta)$ of self-healing FRP specimens with $W_{f}=30 \mathrm{wt} \%$ and $N=6000$ are presented in Fig. (5) as a function of $d$. As shown in Fig. (5), there is a significant amount of scatter in the data between specimens. The scatter may be attributed to the microstructural inhomogeneity of this material and the difficulty of specimen preparation. However, such behavior is characteristic of mechanical testing of FRPs in general. In this study, the

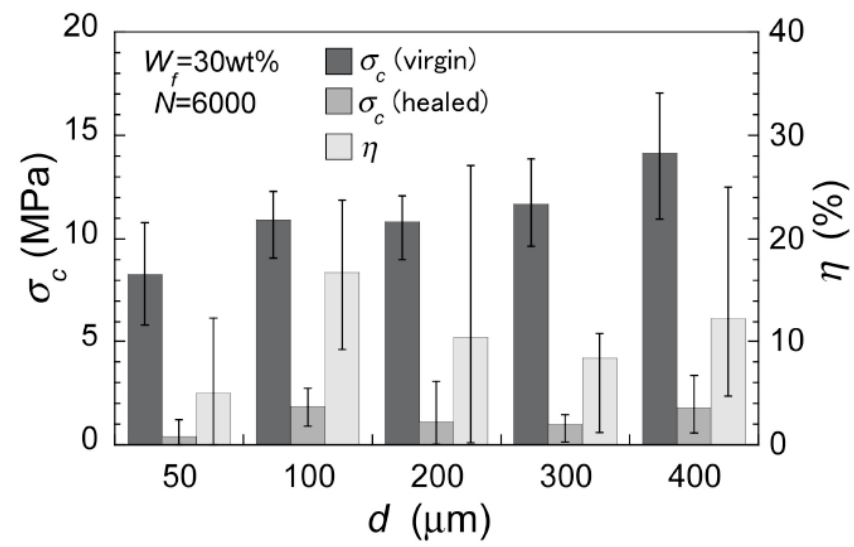

Fig. (5). Virgin and healed tensile strengths, and healing efficiency vs. microcapsule mean diameter. test results for each group of specimens are averaged to see a trend. The $d$ affected the average virgin tensile strength and the average virgin tensile strength of the specimens with $d$ $=400 \mu \mathrm{m}$ was higher as compared to other results. The average healed tensile strength and the average healing efficiency were essentially independent of $d$.

The virgin and healed tensile strengths, and healing efficiency of self-healing FRP specimens with $d=300 \mu \mathrm{m}$ and $N=6000$ are plotted in Fig. (6) as a function of $W_{f}$. For the specimen with $W_{f}=10 \mathrm{wt} \%$, no healing was observed. The virgin and healed tensile strengths were insensitive to $W_{f}$ as the $W_{f}$ increased from 15 to $40 \mathrm{wt} \%$. Moreover, scatter in the virgin tensile strength is dramatically increased at higher concentrations. Large scatter was observed in the data as a result of problems with mixing of the microcapsules and the Grubbs catalyst. The $W_{f}$ slightly affected the average healed tensile strength and the average healing efficiency.

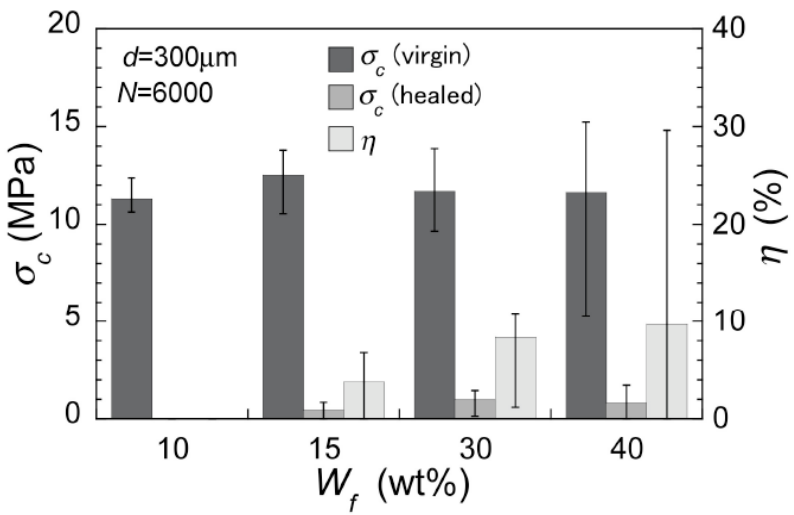

Fig. (6). Virgin and healed tensile strengths, and healing efficiency vs. microcapsule concentration.

Fig. (7) shows the virgin and healed tensile strengths, and healing efficiency of self-healing FRP specimens with $W_{f}$ $=30 \mathrm{wt} \%$ and $d=300 \mu \mathrm{m}$ as a function of $N$. The specimens with $N=6000$ exhibited a high average virgin tensile strength when compared to the specimens with $N=3000$ and 12000 . In contrast, the $N$ did not have a significant effect on the average healed tensile strength. Though the scatter in the data is appreciable, a high average healing efficiency was present at $N=12000$.

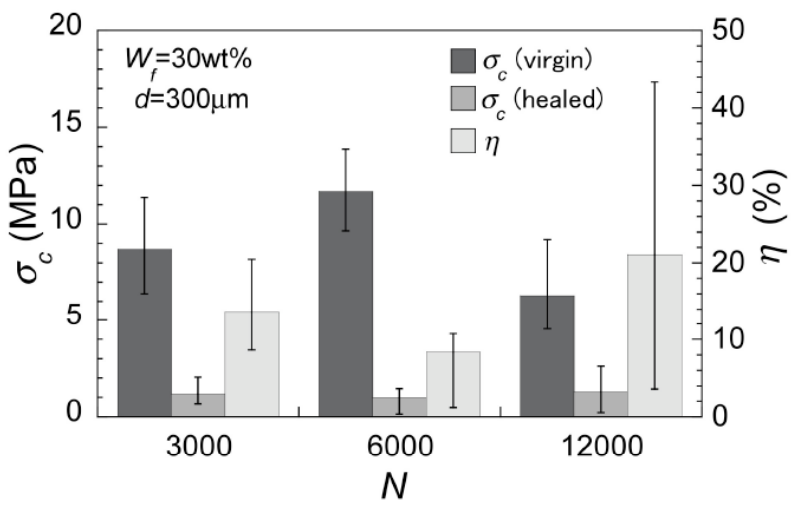

Fig. (7). Virgin and healed tensile strengths, and healing efficiency $v s$. number of filaments in reinforcements.

SEM images of the fracture surface of the healed specimens with $d=50$ and $100 \mu \mathrm{m}$ are shown in Fig. (8). 


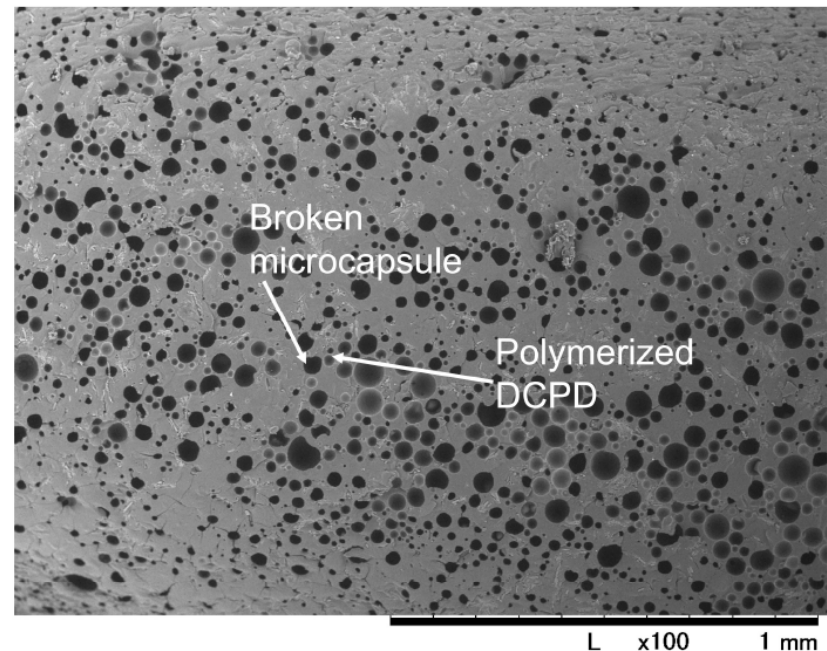

(a)

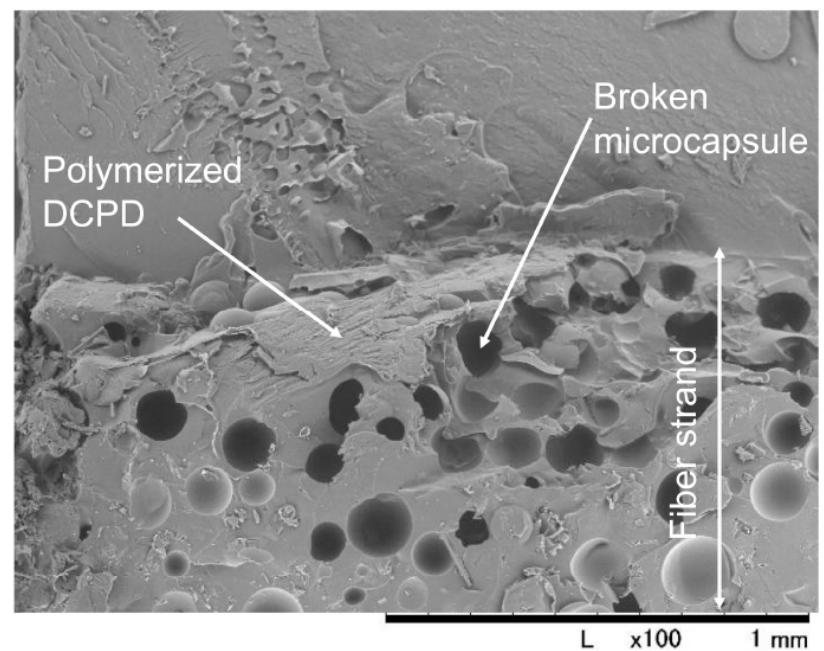

(b)

Fig. (8). SEM micrographs of fracture surface of healed specimens; (a) $d=50 \mu \mathrm{m}$, (b) $d=100 \mu \mathrm{m}$.

For the specimen with $d=50 \mu \mathrm{m}$ (Fig. 8a), there are few observable polymerized healing agents. For the specimen with $d=100 \mu \mathrm{m}$ (Fig. 8b), polymerized healing agent was present in the vicinity of fractured microcapsules.

Fig. (9) presents SEM images of the fracture surfaces of the healed specimens with $W_{f}=10$ and $40 \mathrm{wt} \%$. For the specimen with $W_{f}=10 \mathrm{wt} \%$ (Fig. 9a), broken fiber strands are evident on the fracture plane. For the specimen with $W_{f}$ $=40 \mathrm{wt} \%$ (Fig. 9b), it is clear from the image that crack propagation occurred through the coating layer of the fiber strand surface. The morphology of the healed fracture surface is quite distinct from the specimen with $W_{f}$ $=10 \mathrm{wt} \%$ and many broken microcapsules are evident on the fracture plane.

Fig. (10) shows SEM images of the fracture surface of the healed specimens with $N=3000,6000$ and 12000. In all images (Fig. 10a, b and c), many fractured microcapsules release the encapsulated healing agent. However, there was no detectable correlation between fracture plane morphology and $N$.

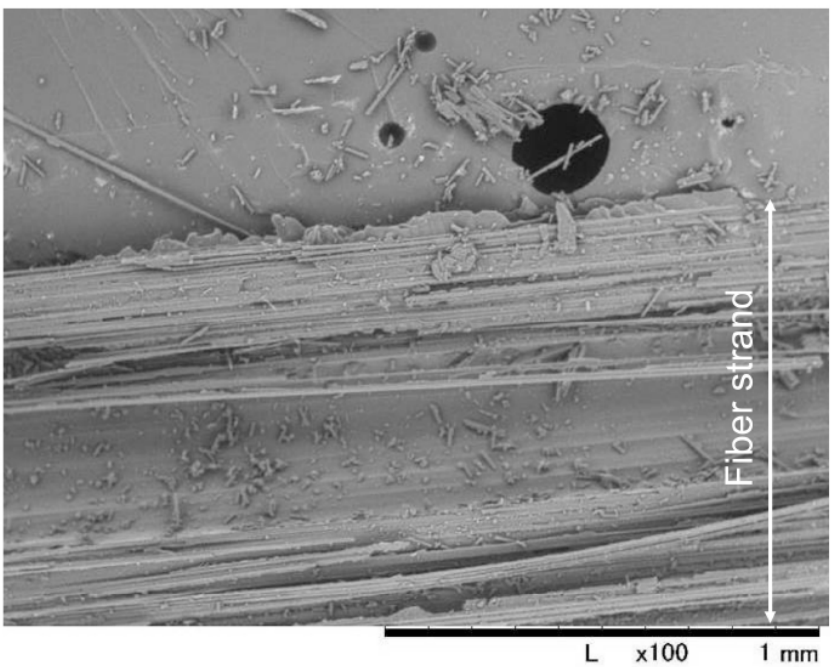

(a)

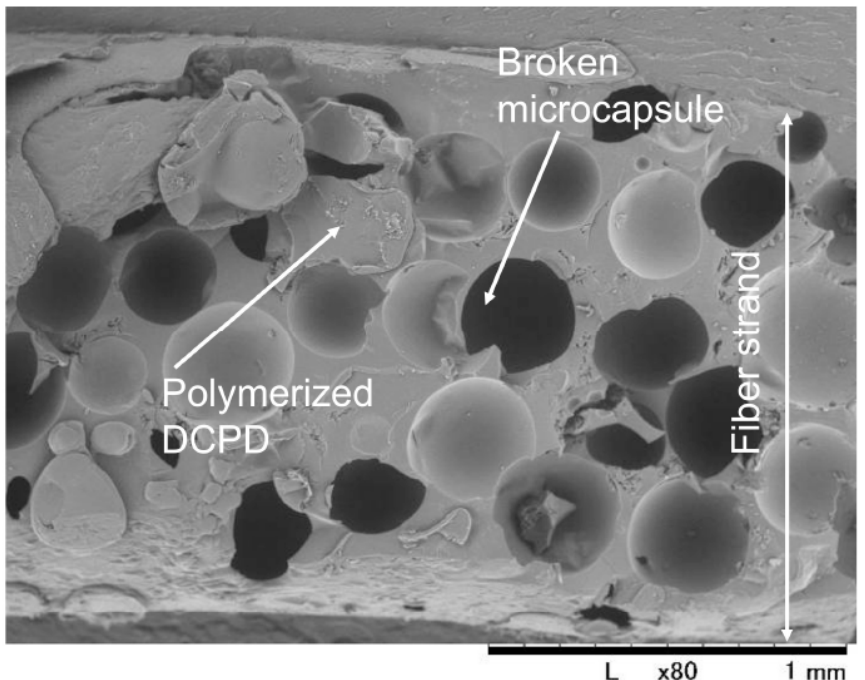

(b)

Fig. (9). SEM micrographs of fracture surface of healed specimens; (a) $W_{f}=10 \mathrm{wt} \%$, (b) $W_{f}=40 \mathrm{wt} \%$.

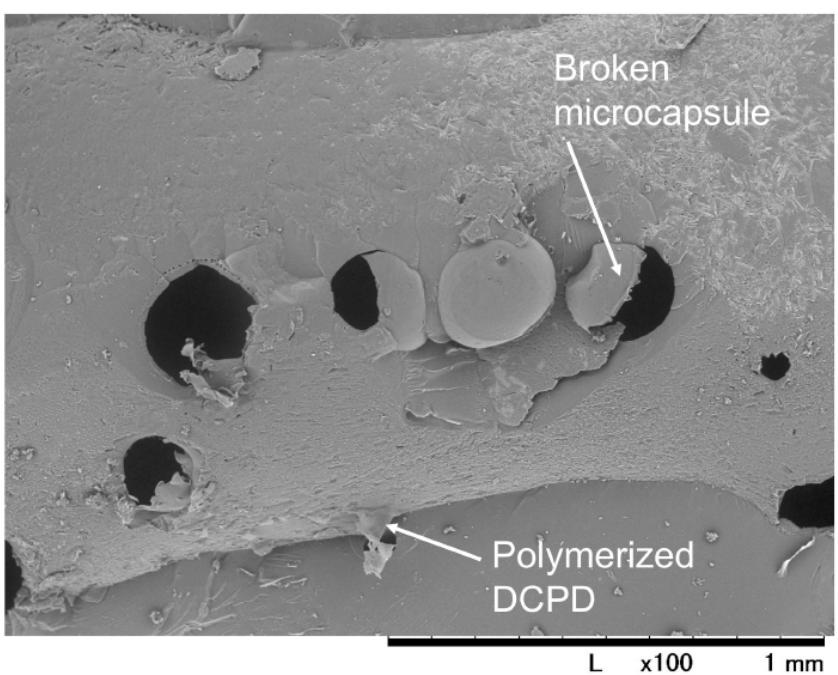

(a) 
(Fig. 10). contd.....

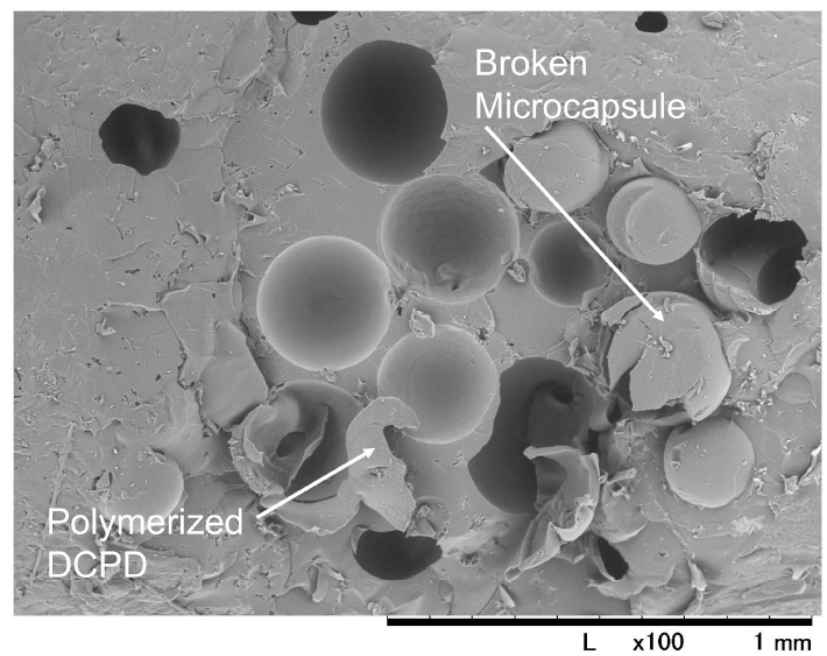

(b)

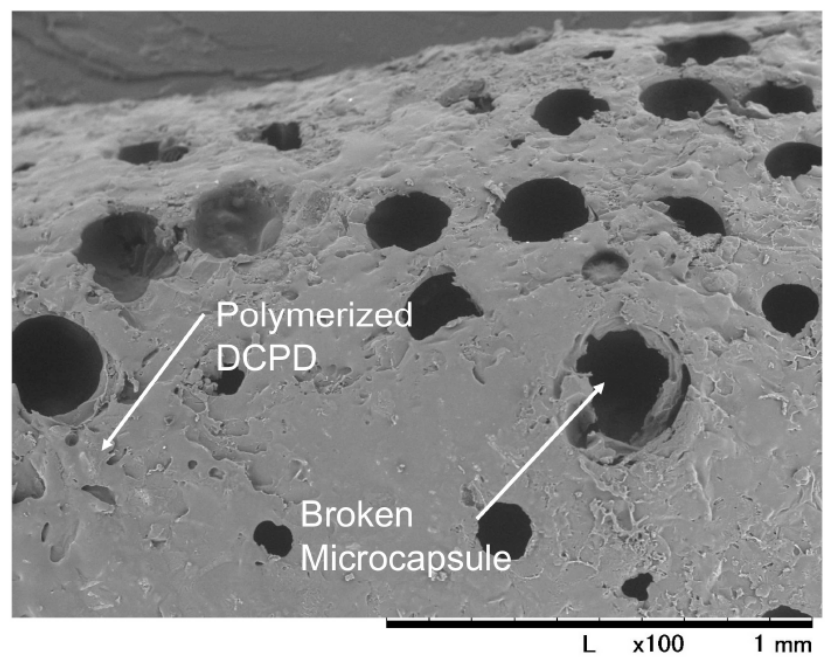

(c)

Fig. (10). SEM micrographs of fracture surface of healed specimens; (a) $N=3000$, (b) $N=6000$, (c) $N=12000$.

\section{Matrix-Microcapsule Debonding Analysis}

Fig. (11) shows the bonding area normalized by surface area of microcapsule, $A / A_{0}$, versus the nondimensional displacement, $u^{*} / L$. Calculation were carried out assuming $V_{f}=0.1, \sigma_{\text {max }}=0.1 \mathrm{MPa}, \delta_{c n}=0.1 \mu \mathrm{m}, p=10 \mathrm{kPa}, 2 R=$ $100 \mu \mathrm{m}$ and $t=0.24 \mu \mathrm{m}$. The debonding between

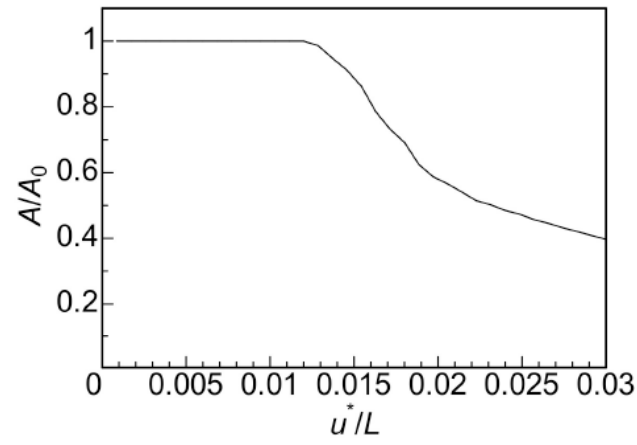

Fig. (11). Typical bonding area vs. displacement curve. microcapsule and matrix initiates when the $u^{*} / L$ reaches about 0.013 . The debonding damage propagates rapidly as the $u^{*} / L$ increases approximately from 0.013 to 0.02 . As the $u^{*} / L$ increases further, the damage growth rate is reduced.

Fig. (12) shows the predicted debonding damage progression in the unit cell model viewed from surface $x_{2}=0$. It can be observed from Fig. (12a) that debonding initiates at the equator. As the $u^{*} / L$ increases further, debonding damage propagates from the equator to the pole Figs. (12b and c).

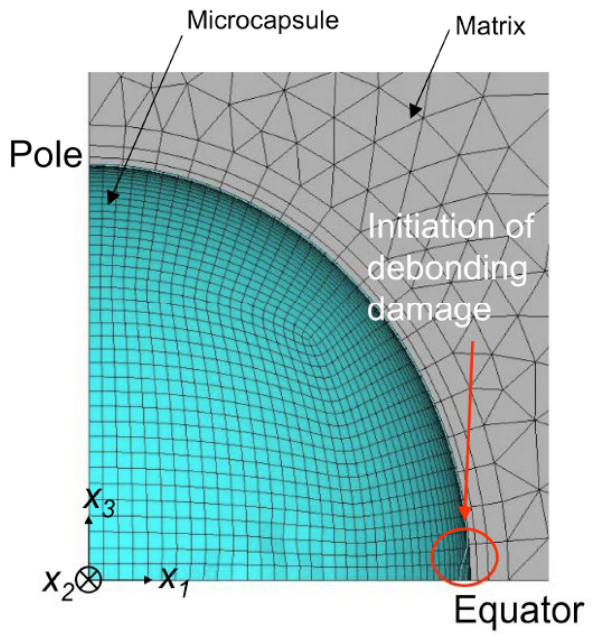

(a)

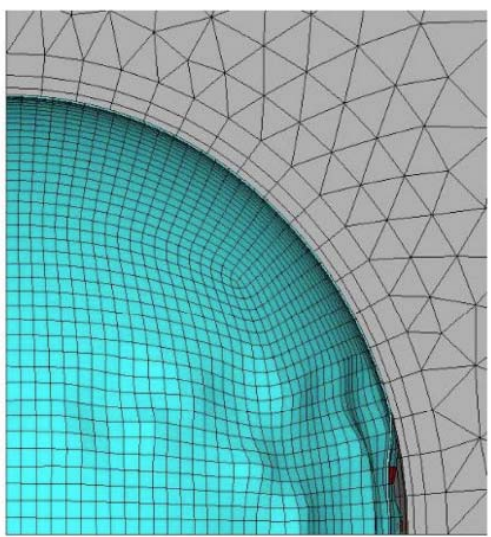

(b)

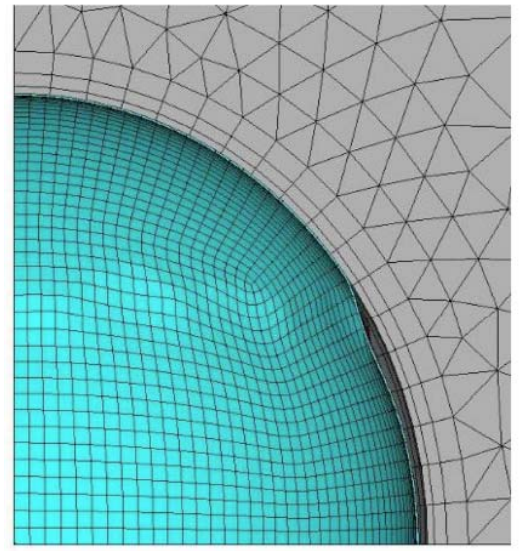

(c)

Fig. (12). Illustration of the predicted debonding damage growth pattern in the unit cell model viewed from surface $x_{2}=0$ as a function of the applied displacement; (a) $u^{*} / L=0.013$, (b) $u^{*} / L=0.018$, (c) $u^{*} / L$ $=0.027$. 
The corresponding results for the unit cell model viewed from surface $x_{3}=0$ are shown in Fig. (13). At $u^{*} / L=0.013$ (Fig. 13a), the debonding damage initiates at surfaces $x_{1}=0$ and $x_{2}=0$. As the $u^{*} / L$ increases further (Fig. $\mathbf{1 3 b}$ and $\mathbf{c})$, the debonding damage becomes larger.

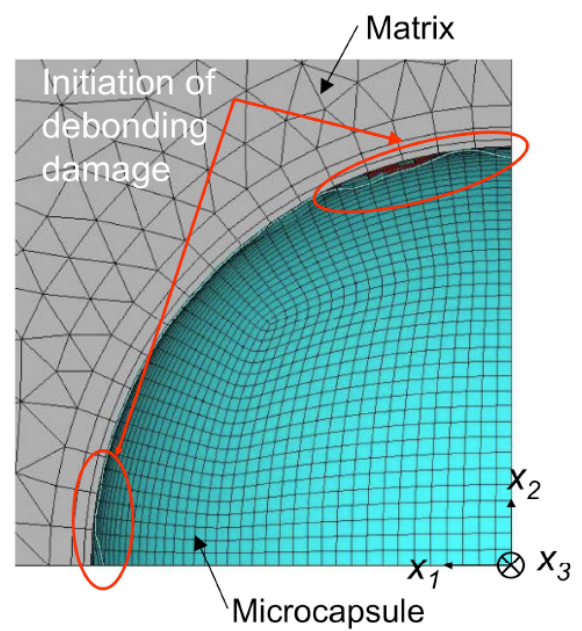

(a)

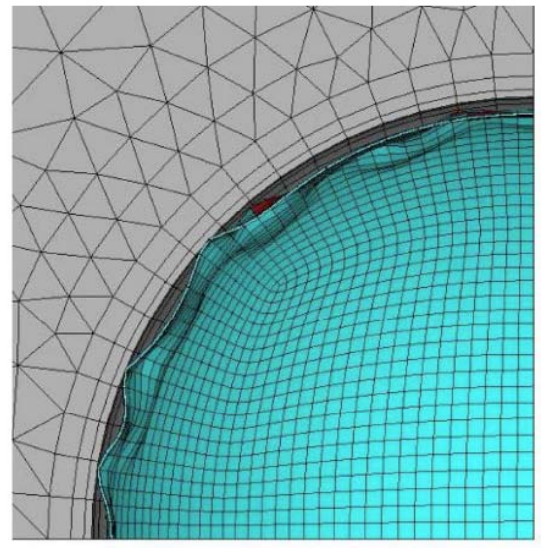

(b)

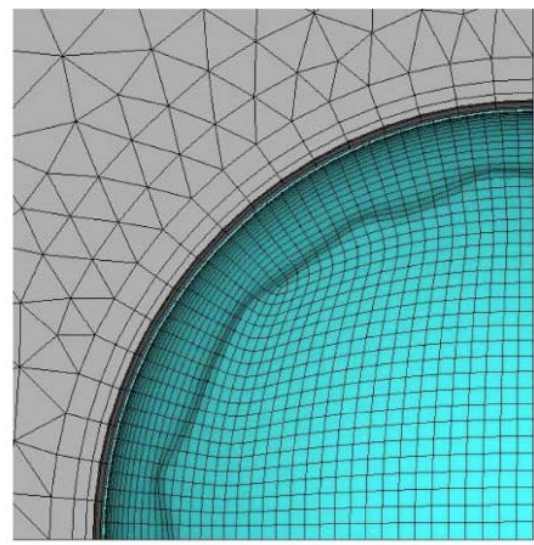

(c)

Fig. (13). Illustration of the predicted debonding damage growth pattern in the unit cell model viewed from surface $x_{3}=0$ as a function of the applied displacement; (a) $u^{*} / L=0.013$, (b) $u^{*} / L$ $=0.018,(\mathbf{c}) u^{*} / L=0.027$.

Fig. (14) shows the influence of $\sigma_{\max }$ on debonding damage behavior. As the $\sigma_{\max }$ decreases, the debonding damage occurs at lower displacement. Fig. (15) provides similar plots for various $\delta_{c n}$. As the $\delta_{c n}$ increases from 0.01

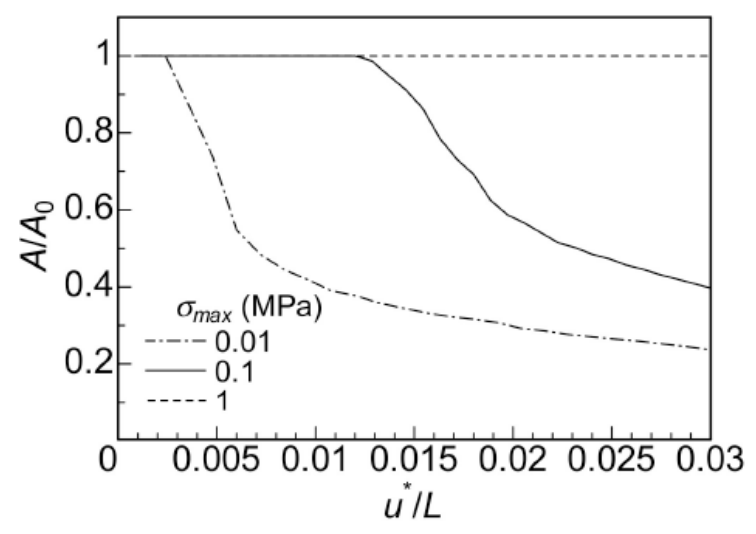

Fig. (14). Effect of interfacial strength on debonding behavior.

to $1 \mu \mathrm{m}$, the debonding damage growth rate decreases. The debonding damage behavior changes from brittle to ductile with increasing $\delta_{c n}$. Fig. (16) exhibits the effect of $2 R$ on the debonding damage behavior. The debonding damage initiates at lower displacement as the $2 R$ decreases. This is similar to the results for the $\sigma_{\max }$. If the debonding damage occurs, the release of healing agent in the microcapsule is believed to be incomplete. The microcapsule diameter is a critical parameter to obtain higher healed strength. The corresponding results for various $V_{f}$ are shown in Fig. (17). The debonding damage behavior is insensitive to $V_{f}$ ranging from 0.01 to 0.2 . The experimental results showed that the average virgin tensile strength decreased with decreasing microcapsule mean diameter. This may be attributed to the microcapsule-matrix debonding damage that occurs during the virgin test. Therefore, the numerical results are useful to interpret the experimental behavior.

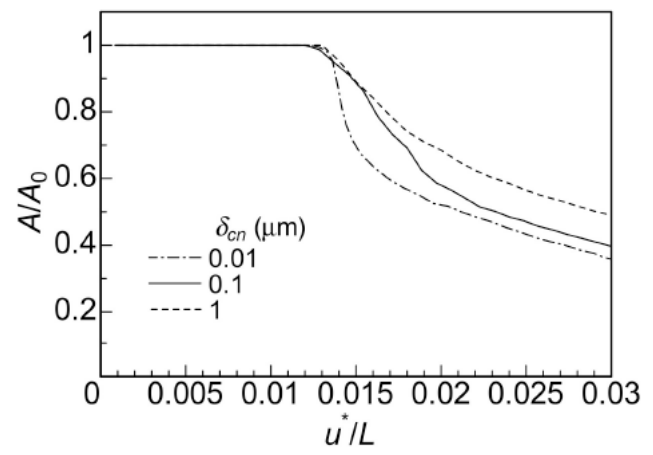

Fig. (15). Effect of critical opening displacement on debonding behavior.

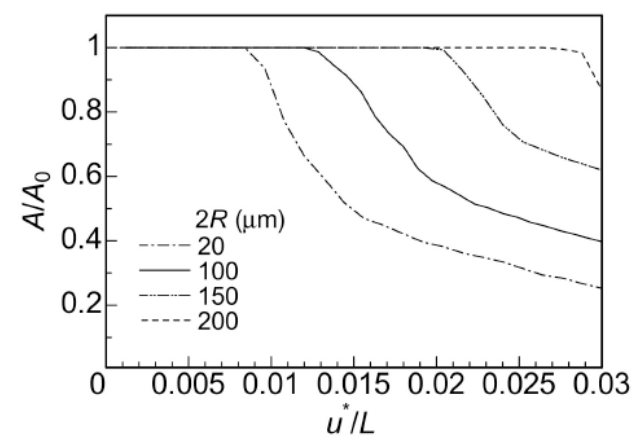

Fig. (16). Effect of microcapsule diameter on debonding behavior. 


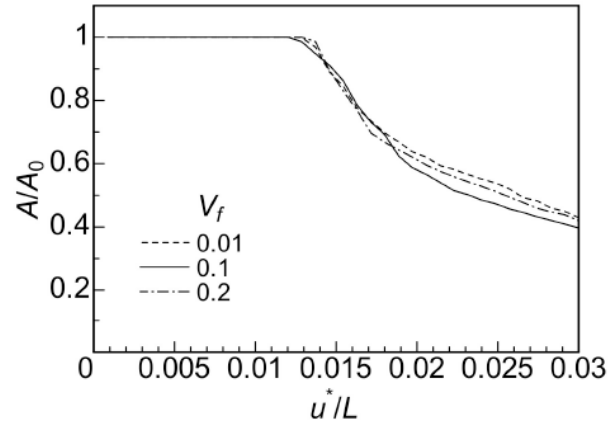

Fig. (17). Effect of microcapsule volume fraction on debonding behavior.

\section{CONCLUSIONS}

The self-healing of interfacial debonding in FRPs and the effect of microstructure on strength recovery were investigated. Transverse tensile tests were conducted using unidirectional FRP. The effects of microcapsule mean diameter and concentration, and number of filaments in the fiber strand on tensile strength of virgin and healed specimens were evaluated. Finite element analyses were also carried out to interpret the debonding behavior of the interface between the microcapsule and the matrix. Based on the study, the following conclusions can be made:

1. The $N$ and $d$ were shown to have an impact on the virgin tensile strength of self-healing FRP. The improvement in the average virgin tensile strength was obtained with $d=400 \mu \mathrm{m}$ and $N=6000$. However, the $W_{f}$ had a negligible effect on the average virgin tensile strength.

2. The effects of $N$ and $d$ on the healed tensile strength and the healing efficiency of self-healing FRP were weak. However, the $W_{f}$ appeared to have little effect on the average healed tensile strength and the average healing efficiency.

3. The debonding damage between the microcapsule and matrix occurred at lower displacement as the microcapsule diameter decreased. This result indicates that higher healing efficiency is obtained for larger microcapsule diameter. Moreover, there is no effect of microcapsule volume fraction ranging from 0.01 to 0.2 on the debonding behavior. Because the reduction in the virgin tensile strength may be attributed to the microcapsule-matrix debonding damage, the proposed model predicted the experimental behavior well.

\section{ACKNOWLEDGEMENTS}

This work was supported by the Ministry of Education, Culture, Sports, Science and Technology of Japan under the Grant-in-Aid for Young Scientists (B), 16760572. The authors gratefully acknowledge the technical advice and guidance of Professor Makoto Kawagoe.

\section{REFERENCES}

[1] S.A. Hayes, W. Zhang, M. Branthwaite, and F.R. Jones, "Self-healing of damage in fibre-reinforced polymer-matrix composites", J. R. Soc. Interface, vol. 4, pp. 381-387, 2007.

[2] R.S. Trask, G.J. Williams, and I.P. Bond, "Bioinspired self-healing of advanced composite structures using hollow glass fibers", J. R. Soc. Interface, vol. 4, pp. 363-371, 2007.

[3] C. Dry, "Procedures developed for self-repair of polymer matrix composite materials", Compos. Struct., vol. 35, pp. 263-269, 1996.

[4] M. Motuku, U.K. Vaidya, and G.M. Janowski, "Parametric studies on self-repairing approaches for resin infused composites subjected to low velocity impact", Smart Mater. Struct., vol. 8, pp. 623-638, 1999.

[5] S.M. Bleay, C.B. Loader, V.J. Hawyes, L. Humberstone, and P.T. Curtis, "A smart repair system for polymer matrix composites", Composites Part A, vol. 32, pp. 1767-1776, 2001.

[6] J.W.C. Pang, and I.P. Bond, "Bleeding composites - damage detection and self-repair using a biomimetic approach", Composites Part A, vol. 36, pp. 183-188, 2005.

[7] S.R. White, N.R. Sottos, P.H. Geubelle, J.S. Moore, M.R. Kessler, S.R. Sriram, E.N. Brown, and S. Viswanathan, "Autonomic healing of polymer composites", Nature, vol. 409, pp. 794-797, 2001.

[8] M.R. Kessler, and S.R. White, "Self-activated healing of delamination damage in woven composites", Composites Part A, vol. 32, pp. 683-699, 2001.

[9] T. Yin, L. Zhou, M.Z. Rong, and M.Q. Zhang, "Self-healing woven glass fabric/epoxy composites with the healant consisting of microencapsulated epoxy and latent curing agent", Smart Mater. Struct., vol. 17, pp. 8, 2008.

[10] K. Sanada, I. Yasuda, and Y. Shindo, "Transverse tensile strength of unidirectional fiber-reinforced polymers and self-healing of interfacial debonding", Plast. Rubber Compos., vol. 35, pp. 67-72, 2006.

[11] F.J. Guild, and A.J. Kinloch, "Predictive modeling of the mechanical properties of rubber-toughened epoxy", J. Mater. Sci. Lett., vol. 13, pp. 629-632, 1994.

[12] G. Alfano, and A. Crisfield, "Finite element interface models for the delamination analysis of laminated composites: Mechanical and computational issues", Int. J. Numer. Methods Eng., vol. 50, pp. 1701-1736, 2001.

(C) Sanada et al.; Licensee Bentham Open.

This is an open access article licensed under the terms of the Creative Commons Attribution Non-Commercial License (http://creativecommons.org/licenses/by$\mathrm{nc} / 3.0 /$ ), which permits unrestricted, non-commercial use, distribution and reproduction in any medium, provided the work is properly cited. 